\section{Mesa de Trabajo Intersectorial en Salud de Santa Fe: una estrategia de participación colectiva para la prevención del dengue}

Hernán Blesio

Universidad Nacional del Litoral, Argentina. hernanblesio@gmail.com

(iD) orcid.org/0000-0001-5234-7557
Sujetos y relaciones en extensión universitaria / Intervenciones
RECEPCIÓN: 01/04/20

ACEPTACIÓN FINAL:16/06/20

\section{Resumen}

La enfermedad del denge representa un problema de salud pública para la región del litoral argentino y para la ciudad de Santa Fe en particular. La Mesa de Trabajo Intersectorial en salud, liderada por el gobierno municipal, incluye a distintos actores sociales para intentar encontrar soluciones frente a esta problemática.

La Facultad de Ciencias Médicas de la Universidad Nacional del Litoral participa activamente de esta Mesa, cuestión que le permite promover el vínculo con actores del territorio, poner en juego contenidos académicos, dialogar con otros saberes y formar profesionales con un perfil en Atención Primaria de la Salud.

El artículo se propone analizar las relaciones de los sujetos que participaron de las campañas de prevención del dengue en el año 2019 , sus roles, intereses, fortalezas y debilidades en el trabajo en territorio, el impacto de la intervención en los mismos y de qué manera estas actividades se presentan como una oportunidad para el desarrollo de contenidos de diversas disciplinas de la carrera de Medicina.

Palabras clave: Mesa de Trabajo Intersectorial de salud; políticas públicas en salud; prevención del dengue; gobiernos locales; salud comunitaria; extensión universitaria
Santa Fe Intersectoral Health Group: a collective participation strategy for dengue prevention

\section{Abstract}

UNL's School of Medical Sciences takes part in the "Health Intersectoral Board" (or MTI, in Spanish) of Santa Fe City to encourage the bond with the different parts of the territory and to introduce a place to address content, and which also allows the exchange of knowledge and the development of professionals with a Primary Health Care profile.

The MTI promotes the involvement of the community in public policies. This strengthens the ability to detect territory problems, and to think and carry out actions that bring solutions and tools for people to take care of themselves and improve their health.

In this context, dismissal campaigns were coordinated in the city's neighborhoods that were mostly affected by dengue during 2019 in order to prevent the disease.

The article aims to analyze the relationships of the subjects who participated in the dengue prevention campaigns in 2019, their roles, interests, strengths and weaknesses working in the territory, the impact of the intervention on them and in what way these activities are presented as an opportunity to develop content from various disciplines of the Medicine career.

Keywords: Intersectoral Board of Health; public health policies; dengue prevention; local governments; community health; universtity extension
Mesa de Trabalho Intersetorial de Saúde de Santa Fe: uma estratégia de participação coletiva na prevenção contra dengue

\section{Resumo}

A doença da dengue representa um problema de saúde pública para a região do litoral argentino e para, particularmente, a cidade de Santa Fe. A Mesa de Trabalho Intersetorial liderada pelo governo municipal inclui diversos atores sociais para tentar encontrar soluções frente à problemática.

A Faculdade de Ciências Médicas da Universidade Nacional do Litoral participa ativamente nesta Mesa, questão que lhe permite promover o vínculo com atores do território, pôr em jogo conteúdos acadêmicos, dialogar com outros saberes e formar profissionais com um perfil em Atenção Primária à Saúde.

$\mathrm{O}$ artigo se propõe analisar as relações dos sujeitos que participaram nas campanhas de prevenção contra a dengue no ano de 2019, as suas funções, os seus interesses, as suas fortalezas e as suas fraquezas no trabalho em território, o impacto da intervenção neles e o jeito em que estas atividades se apresentam como uma oportunidade para o desenvolvimento de conteúdos de diversas disciplinas do curso de Medicina.

Palavras-chave: Junta Intersetorial de Saúde; políticas de saúde pública; prevenção contra dengue; governos locais; saúde comunitária; extensão universitária 


\section{Introducción}

Las políticas públicas en salud requieren de la participación de diferentes actores sociales y niveles del Estado para conformar redes con la capacidad de atenuar el impacto de los determinantes sociales de la salud y, con ello, las inequidades sanitarias.

La Facultad de Ciencias Médicas (FCM) de la Universidad Nacional del Litoral (UNL) se posicionó como un actor estratégico en el área de salud de la región por su trabajo en docencia e investigación, sumado a la presencia territorial que le genera la incorporación de la extensión en sus carreras desde los primeros años. Esto posibilitó que desde 2018 forme parte de la Mesa de Trabajo Intersectorial de salud (MTI) impulsada desde el gobierno de la ciudad de Santa Fe.

Según las recomendaciones de la Organización Mundial de la Salud (OMS), es fundamental el rol de los gobiernos locales debido a su cercanía a las personas y presencia en el territorio como generador de instancias de participación de los actores sociales en su conjunto. La universidad aporta en estos espacios el conocimiento científico y técnico con sus equipos docentes y un recurso humano en formación, como son los estudiantes. Estos recursos sirven para pensar y llevar adelante estrategias para abordar problemáticas sociales. Tanto el gobierno como la universidad, sumados a instituciones no gubernamentales con anclaje territorial, forman un equipo con diferentes saberes, construidos por diversas trayectorias, y que son puestos en común para brindar mejores respuestas a los problemas de salud que se surgen en el territorio.

Es ahí donde una MTI en salud se presenta como una importante herramienta para el vínculo de los actores a la hora de realizar análisis de la situación de salud de un barrio, una ciudad o una región. Este espacio funciona como una institución política que arma agenda, planifica, ejecuta y evalúa políticas de promoción de la salud y prevención de la enfermedad.

Los estudiantes universitarios y los equipos docentes encuentran en estos espacios una oportunidad para desarrollar contenidos curriculares en un contexto real que permiten consolidar una formación para profesionales del primer nivel de atención, respondiendo a estándares planteados por la OMS.

En la experiencia que se narra trabajaron estudiantes de la carrera de Medicina con docentes de las áreas de Injuria, Clínica Médica, Práctica Final Obligatoria, correspondientes a los ciclos superiores, y de la disciplina Salud Pública, que se desarrolla de manera transversal en todos los ciclos del plan de estudio.

El texto explica por qué la práctica de la extensión universitaria en la FCM implica un campo de relaciones de los sujetos, la forma en que se vinculan estos actores y cómo estas relaciones se dan por temáticas o problemas de salud que se identifican como prioritarios o importantes para la academia. Además, describe los modos en que equipos de la Facultad elaboran la agenda de trabajo y analizan y evalúan políticas públicas que atraviesan la problemática escogida.

Las intervenciones realizadas en la ciudad de Santa Fe en 2019, con el objetivo de prevenir la enfermedad del dengue, dan cuenta de cómo, desde un espacio institucional como es la MTI, la FCM intercambia saberes y trabaja con organizaciones de la sociedad civil, instituciones públicas, movimientos sociales de base territorial y con ciudadanos interesados o grupos de vecinos en pos de aportar soluciones a los problemas sociales que impactan en la salud individual y de la comunidad. 
Se detalla, además, en el marco de la intervención, desde qué paradigmas se piensa la extensión universitaria y desde qué estrategias los equipos docentes trabajan para abordar los contenidos curriculares en los diversos escenarios que le ofrece la Facultad al formar parte de una MTI en salud.

En una primera parte, el artículo hace un recorrido por la historia que tiene la enfermedad en la región y cómo esta se constituye como un problema para la salud pública. Luego se describe desde qué lugar se piensa a la salud y, con ello, las herramientas que tienen las comunidades para garantizar la participación social. El texto continúa haciendo un desarrollo de los paradigmas en que se piensa la extensión en la FCM y la importancia de la misma en la formación de los profesionales de la salud. A continuación, se narra la experiencia de la intervención en materia de prevención de la enfermedad del dengue y se hace un análisis del impacto en los diferentes actores; se realiza una valoración de dicha intervención haciendo foco en las fortalezas y debilidades y en las líneas futuras de trabajo.

\section{El dengue, un problema de salud pública en la región}

El dengue es una enfermedad viral transmitida por el mosquito Aedes aegypti:

"Se trata de un problema creciente para la salud pública mundial debido a varios factores como el cambio climático, el aumento de la población mundial en áreas urbanas de manera rápida y desorganizada, la insuficiente provisión de agua potable que obliga al almacenamiento de la misma en recipientes caseros habitualmente descubiertos, la inadecuada recolección de residuos y la gran producción de recipientes descartables y neumáticos desechados que sirven como criaderos de mosquitos. A estos factores se suman el aumento de los viajes y las migraciones, y el control insuficiente de los vectores". (Ministerio de Salud de la Provincia de Santa Fe, 26 de noviembre de 2013, s/p.)

Los sitios de cría del mosquito son fundamentalmente artificiales: urbanos (en baldíos, cementerios, desarmaderos, basurales) o domésticos (neumáticos, floreros, botellas, bebederos de animales, latas abiertas o contenedores de cualquier tipo, depósitos de agua de bebidas, cisternas, vasijas, tinajas, todo tipo de recipientes en desuso).

El ciclo evolutivo del Aedes aegypti se inactiva con las bajas temperaturas, pero los huevos pueden permanecer en estado latente por largos períodos de tiempo, hasta que se den nuevamente las condiciones óptimas de temperatura y humedad que le permiten eclosionar y de esta manera completar el ciclo hasta tener un mosquito adulto. Esto sucede generalmente en primavera. De este modo, si la hembra que puso huevos estaba infectada de dengue, existen probabilidades de trasmitir el virus al huevo y, en consecuencia, tener mosquitos adultos infectados.

Por lo tanto, la eliminación de objetos innecesarios que juntan agua y el correcto manejo de los que resultan de utilidad, en domicilios y espacios comunes dentro de la comunidad, resulta una actividad fundamental en el control de la población del vector.

Se denomina descacharrado o descacharrizado a la eliminación de todos los objetos inservibles que se encuentran en el domicilio, en el peridomicilio (alrededor de la vivienda) y en la comunidad, que pueden acumular agua y servir de este modo como criaderos del mosquito Aedes aegypti. 
La ciudad de Santa Fe se encuentra asentada entre los valles de inundación de dos ríos de llanura, el Paraná y el Salado. Estos, con sus ciclos de crecidas y bajantes periódicas, sumado a su clima húmedo, subtropical, con las cuatro estaciones bien definidas, dan un marco propicio para la existencia del vector.

Según informes de la Sala de Situación de Salud Local, la provincia de Santa Fe registró a comienzos del año 2009 su primer brote de dengue, y su segundo brote fue en 2011, con casos autóctonos. Particularmente, en la ciudad de Santa Fe se registró en 2016 más de 290 casos. Durante 2019 se identificaron brotes en distintos barrios de nuestra localidad, contabilizando más de 300 casos de dengue.

La Municipalidad de la ciudad cuenta con protocolos, dentro del marco de gestión de riesgo, que le permiten actuar de manera ordenada con equipos capacitados propios, de diferentes áreas; y desde hace mucho tiempo se vincula con instituciones como la UNL, Universidad Tecnológica Nacional - Facultad Regional Santa Fe, la Cruz Roja Argentina, la Sociedad Argentina de Pediatría filial Santa Fe, la Federación Universitaria del Litoral y distintos sectores del gobierno provincial, vecinales, centros de jubilados y clubes barriales, para dar respuesta a estos problemas sanitarios y trabajar fuertemente en la prevención de enfermedades transmitidas por mosquitos, entre otras temáticas.

\section{¿Desde qué paradigmas pensamos la salud?}

La definición de salud de la OMS resalta una mirada holística y nos obliga a pensarla como un proceso complejo y dinámico en el que intervienen muchos factores en su determinación. El concepto de determinantes de la salud fue acuñado por Marc Lalonde en 1974 y se complejizó con el paso de los años hasta llegar en la actualidad a la definición que de ellos hace la OMS:

"son las circunstancias en que las personas nacen, crecen, viven, trabajan y envejecen, incluido el sistema de salud. Esas circunstancias son el resultado de la distribución del dinero, el poder y los recursos, que dependen a su vez de las políticas adoptadas". (s/f)

Estos determinantes son los que explican las inequidades sanitarias, esas diferencias injustas que obligan a los actores del ámbito de la salud a analizar, planificar, ejecutar y reflexionar en conjunto.

Entre los determinantes estructurales se encuentra el contexto socioeconómico y político, que se refiere a los factores que afectan de manera importante a la estratificación social y la distribución de poder y recursos dentro de ella. Los distintos ejes de desigualdad, como son la clase social, el género, la edad, la etnia o la raza, y el territorio de procedencia o de residencia, establecen jerarquías de poder en la sociedad que repercuten en las oportunidades de tener una buena salud a través de la exposición a los llamados determinantes intermedios. Estos últimos incluyen los recursos materiales, como son las condiciones de empleo (situación laboral, precariedad) y de trabajo (riesgos físicos y ergonómicos, organización y entorno psicosocial), la carga de trabajo no remunerado del hogar y de cuidado de las personas, el nivel de ingresos y la situación económica y patrimonial, la calidad de la vivienda y sus equipamientos, el barrio o área de residencia y sus características. Estos recursos, junto con la posición de poder, tienen un impacto en la salud tanto directo como indirecto e influyen en procesos psicosociales, tales como la falta de control, la autorrealización, las situaciones 
de estrés, y en las conductas individuales. Finalmente, aunque los servicios sanitarios en sí mismos no constituyen el factor principal de generación de las desigualdades en salud, pueden tener un efecto tanto multiplicador como amortiguador de la desigualdad, dependiendo de si su acceso, utilización y calidad son menores, iguales o mayores (respecto de la misma necesidad) para los grupos sociales menos favorecidos.

El proceso de salud y de enfermedad de las personas requiere del compromiso de los Estados en todos sus niveles y en sus diferentes sectores, pero también es fundamental el de las organizaciones no gubernamentales que integran redes en el territorio. La participación individual o colectiva en la planificación de políticas de salud es un derecho y un deber del pueblo y el Estado debe generar los espacios y las herramientas para hacerla posible.

Entender la salud como un proceso complejo que se construye entre todos supone considerar que las acciones intersectoriales constituyen un recurso fundamental para la solución de los problemas de salud y para el bienestar de la comunidad (Baró, s/f).

En Promoción de Salud, Glosario, la OMS (1998) define la comunidad como un

"grupo específico de personas que a menudo viven en una zona geografía definida, comparten la misma cultura, valores y normas, y están organizadas en una estructura social conforme al tipo de relaciones que la comunidad ha desarrollado a lo largo del tiempo. Los miembros de una comunidad adquieren su identidad personal y social al compartir creencias, valores y normas que la comunidad ha desarrollado en el pasado y que pueden modificarse en el futuro. Sus miembros tienen conciencia de su identidad como grupo y comparten necesidades comunes y el compromiso de satisfacerlas". (s/p)

Jaime y Rosa Gofin (2012) plantean la salud comunitaria como la

"expresión colectiva de la salud de los individuos y grupos en una comunidad definida, determinada por la interacción entre las características de los individuos, las familias, el medio social, cultural y ambiental, así como por los servicios de salud y la influencia de factores sociales, políticos y globales". (p.7)

La participación comunitaria implica una dinámica mediante la cual los ciudadanos se involucran en los procesos que les afectan directa o indirectamente. Esto abre espacios de encuentro entre los ciudadanos y sus gobiernos y posibilita el desarrollo de políticas públicas altamente relacionadas con las expectativas y necesidades de la sociedad.

"En estas dinámicas se crean y comparten percepciones, conocimientos e información, proporcionando así, un sentido de pertenencia. Esto implica una concepción de participación basada en la existencia de ciudadanos activos, informados, motivados, provistos de herramientas para discutir las cuestiones públicas, sus deseos y necesidades, tomar decisiones y acordar a una voluntad colectiva que involucre las diferentes posturas individuales". (Ministerio de Salud de la Nación, diciembre de 2014, p. 12)

Silvana Baró (s/f) plantea la importancia de la intervención de los municipios o comunas en salud ya que son los que conocen a las personas debido a su cercanía. Por ello, cuando un gobierno local pone en agenda la promoción de la salud, en línea a lo que expresa la OMS en la declaración de Alma Ata sobre la Atención Primaria de la Salud (APS), otorga una acción revitalizadora a su función. 
La autora sostiene además que el territorio es el lugar donde se articulan las políticas públicas, es donde surgen los problemas y las soluciones. En ese territorio funcionan redes de actores que, a diferentes escalas, se relacionan de una manera dinámica. Fortalecer la integración de distintos actores consolida las redes en salud que permiten atenuar el impacto de esos determinantes sociales. Es una tarea del municipio abonar a que esto suceda y una MTI es una herramienta facilitadora de esos procesos.

En este sentido, para la universidad, la MTI y el territorio plantean un escenario diferente del aula, donde los docentes y estudiantes pueden abordar los contenidos que plantean los planes de estudio de carreras relacionadas con la salud. En estos espacios se pone en práctica constantemente el trabajo en equipo, intersectorial e interdisciplinariamente, con un enfoque a la salud desde el primer nivel de atención, haciendo foco en la promoción de la salud y prevención de la enfermedad. Todos estos son elementos fundamentales, según la OMS-Organización Panamericana de la Salud (OPS) en el perfil de un trabajador sanitario.

Para los equipos universitarios, integrar esta Mesa requiere de planificación. Como plantea Mario Rovere (2006), planificar es pensar antes de actuar y es un recurso que atañe al área de salud. La planificación es la articulación entre el conocimiento y la acción y es posible mejorarla y pensarla de manera estratégica con instancias de reflexión, sistematización del pensar y aprendizaje en la práctica.

\section{Las Prácticas de Extensión de Educación Experiencial (PEEE) en la FCM}

La extensión universitaria es parte sustancial de la vida de la UNL. Dispositivos institucionales, como programas, proyectos, cursos y PEEE le dan un marco a instancias concretas que permiten a los estudiantes aprender en el contexto real, donde se integra el conocimiento académico-científico con otros saberes.

"Las PEEE son una oportunidad para que la formación universitaria establezca una relación dialéctica entre pensamiento y contexto y para que se desplace, aunque sea solo por un momento, de la encerrona cognitiva que se produce cuando los contenidos disciplinares se ofrecen únicamente en el espacio físico del aula. El proceso de construcción de conocimientos se amplifica y enriquece cuando ocurre mediante la participación de los sujetos en actividades cotidianas y en contextos reales". (Rafaghelli, 2016, p. 10)

Los estudiantes de la FCM tienen la extensión incorporada a la currícula, de modo de promover la articulación y la integración de conocimientos y posibilitar el abordaje de problemas concretos y reales. Esta metodología incluye el diseño de actividades que permiten a los estudiantes hacer análisis de situación, problematizar y pensar estrategias aplicando conocimientos, habilidades y destrezas. Como plantea Rafaghelli (2016), se incorpora el concepto de aprendizaje como proceso y resultado de la participación social y otra idea de estudiante como actor social que aprende mediante la acción en entornos culturales y resuelve problemas reales de la vida.

La UNL tiene líneas de trabajo en relación con enfermedades transmitidas por mosquitos: equipos docentes de diversas casas de estudios desarrollan desde hace años investigación y extensión en la temática. La FCM, en el marco del Programa de Extensión Equidad en Salud, dependiente de la Secretaría de Extensión Social y Cultural de la UNL, 
conformó equipos interdisciplinarios para llevar adelante proyectos de extensión con el objetivo de trabajar en la prevención de dengue, zika y chikunguña, generando así un vínculo, desde hace varios años, con gobiernos locales, vecinales, centros de jubilados, Cruz Roja, Centros de APS, entre otras instituciones. Estos proyectos brindaron espacios para que los estudiantes y docentes experimentaran un proceso de aprendizaje en situación a partir de un determinado tipo de intervención social sobre problemas concretos (Menéndez y Tarabella, 2016).

Esta trayectoria institucional colaboró en cuanto a posicionar a la FCM como referente en temas vinculados a la salud comunitaria, permitiéndole integrar equipos de trabajo interinstitucionales en pos de aportar a la calidad de vida de los ciudadanos de la región. La historia de trabajo en conjunto con el gobierno de la ciudad de Santa Fe se formalizó en 2018 con la propuesta del gobierno local a la FCM de integrar la MTI de salud local.

La Secretaría de Extensión de la Facultad es la que participa de la MTI y vincula la agenda pública. Esta condición posibilita orientar convocatorias específicas a equipos docentes y voluntariados. A su vez, estos equipos docentes brindan a la MTI una lectura técnica y dan respuestas a través de proyectos de extensión o PEEE vinculadas al desarrollo de las actividades académicas que marca el plan de estudio de las diferentes carreras.

Una inquietud de un equipo docente que se da en el marco de la catedra puede ser motivo de la creación de instancias de diálogo con la MTI y con sus actores. La Secretaría de Extensión de la Facultad es la encargada de garantizar esos canales de comunicación que generan un vínculo y desde allí se trabaja. Aunque el diálogo puede darse en otro sentido, como, por ejemplo, un problema social puede ser transmitido por otro actor de la MTI, se discute en marco de la mesa y se convoca a equipos técnicos de la Facultad con el fin de incorporar sus conceptos al debate de las posibles soluciones. Este último ejemplo fue lo originó esta intervención y motivó la conformación de equipos multidisciplinarios.

En tanto, entendemos la multidisciplinariedad en el sentido en que la expresan Díaz Quiñones, Valdés Gómez y Boullosa Torrecilla (2016), como una:

"forma de aproximación al conocimiento, que conduce a la cooperación entre disciplinas que tienen algo en común y que existen hechos, fenómenos, procesos, acciones, elementos que son objetos de estudio de diferentes disciplinas y desde cada disciplina hay demarcación, división especialización y cada una actuando presupone la multidisciplinariedad". (p. 214)

Estos autores también señalan a la interdisciplina como "la revelación de algo que se encuentra en la frontera común a varias disciplinas; mientras que la transdisciplinariedad se refiere a la fundamentación o explicación de las disciplinas desde ese algo común" (p. 214).

Estas definiciones pensadas en el área de salud, teniendo en cuenta sus múltiples determinantes, obligan a conformar equipos de trabajo con estas características, sobre todo cuando deben dar respuestas ante situaciones particulares, como lo es un brote de dengue.

El Programa de Extensión de Equidad en Salud cuenta con voluntarios de la FCM que año a año se inscriben para participar de diversas actividades que se plantean en este marco institucional. Estos alumnos resultan un recurso interesante para trabajar en las intervenciones comunitarias. Entre las temáticas que habitualmente abordan se encuentran las enfermedades transmitidas por mosquitos, por ello fueron convocados junto a estudiantes que 
participaron como voluntarios en proyectos de extensión para la prevención del dengue que llevaron adelante equipos de la Facultad.

Espacios como la MTI y las actividades que de ella se desprenden en materia de prevención del dengue permiten a los estudiantes poner en juego conocimientos teóricos aprendidos en el contexto del aula que van desde la epidemiologia, la estadística, las políticas públicas de salud, el sistema de salud argentino, la APS como estrategia sanitaria, los determinantes sociales de la salud, la comunicación, el paciente con síndrome febril, sus diagnósticos diferenciales, abordajes terapéuticos, estrategias de contención de la enfermedad, protocolos de actuación, responsabilidades del equipo de salud, del Estado, las acciones comunitarias que posibilitan el bloqueo de la enfermedad y sus medidas preventivas, entre otros contenidos que se abordan en un escenario real, con la dinámica y la superposición de saberes que se produce en el territorio. Esto permite luego, desde el aula, resignificar los saberes, discutir sobre la experiencia y producir más y mejores herramientas para su comprensión y su puesta en práctica en la futura vida profesional.

Estas prácticas de extensión se presentan como una oportunidad para el desarrollo de la docencia, pero también habilitan a los equipos de las diferentes disciplinas relacionadas con la salud a generar líneas de investigaciones orientadas a problemas sociales concretos.

\section{La experiencia del trabajo}

A comienzos de 2019, la MTI adquirió en el contexto del brote de dengue una dinámica de trabajo que consistía en reuniones periódicas para evaluar el estado de situación, pensar estrategias de trabajo y sus formas de implementación. También se dieron instancias de reflexión sobre experiencias previas.

Una vez finalizado el brote que sufrió la ciudad el primer semestre de 2019 (ver Figura 1), definió trabajar los meses de frío en acciones de saneamiento y descacharrado asistido en barrios que registraron la mayor cantidad de casos positivos.

Se seleccionaron para eso 220 manzanas de la ciudad que fueron críticas respecto de los casos positivos de dengue registrados en los meses de enero a junio de 2019 y se trabajó con equipos multidisciplinarios e interinstitucionales que fueron capacitados previamente. Los barrios de la ciudad intervenidos fueron: Chalet, Solidaridad y Progreso San Lorenzo y Fomento 9 de Julio (ver Figura 2).

La intervención consistió en realizar colaborativamente con los vecinos el descacharrado y la eliminación de objetos inservibles que resultaran potenciales criaderos del mosquitos; identificar zonas de riesgo dentro de cada vecindad para su posterior limpieza; clasificar los recipientes a descacharrar en las zonas de riesgo para obtener información específica sobre las distintas problemáticas que puedan presentarse en cada área, determinar su disposición final correspondiente y dar herramientas para promover el autocuidado y prevenir enfermedades transmitidas por vectores en espacios comunitarios. Participaron de estas acciones agentes municipales, estudiantes de la FCM, voluntarios de Cruz Roja, equipos de centros de salud, vecinales, Ejército y Gendarmería, entre otros. 


\section{Figura 1. Densidad de casos de dengue en la ciudad de Santa Fe durante el año 2019}

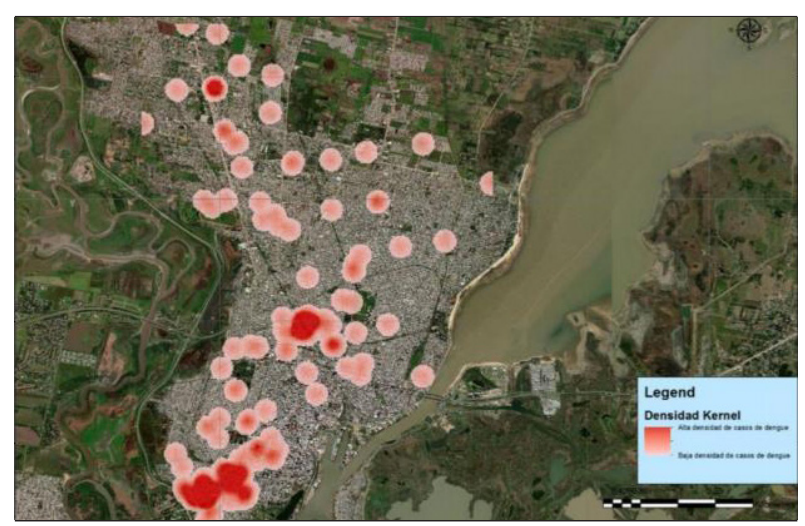

Fuente: Elaboración propia
Figura 2. Ubicaciones de los barrios en los que se realizaron las intervenciones

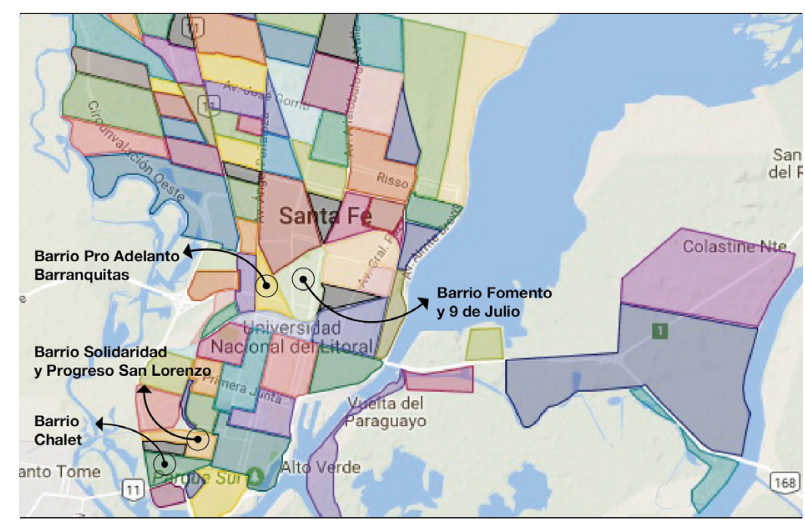

Fuente: http://www.redescasa.com.ar/wp-content/uploads/2017/05/ MAPA-DE-VECINALES-santa-fe.jpg

En ese marco, se efectuaron intensas tareas de limpieza y descacharrado asistido en los meses de julio a septiembre, previo a la eclosión de los huevos que pudieran estar en los recipientes a descartar. Las intervenciones se informaron con antelación mediante diversas estrategias de comunicación (audiovisuales y graficas) que garantizaran la seguridad del vecino y de los equipos y que permitieran un trabajo de mayor calidad en el territorio.

Todas las actividades que se implementaron en materia de limpieza y descacharrado estuvieron acompañadas por acciones educativas con el objetivo de cambiar conductas que ayuden a prevenir las enfermedades transmitidas por mosquitos, pero sobre todo para que sean herramientas que permitan a los vecinos tener un mayor control y protección de su la salud y, con ello, una mejor calidad de vida.

Una aplicación para dispositivos móviles permitió recolectar, ordenar y sistematizar información sobre el número de domicilios intervenidos, puntos peligrosos en relación con la cría de mosquitos, entrega de elementos de protección, etc. Estos datos fueron procesados por la Sala de situación de salud local, que emite su informe periódico a la MTI para su correspondiente análisis.

La metodología de trabajo fue adquiriendo particularidades en los diferentes barrios, de acuerdo con los diversos escenarios planteados en el territorio. Estas singularidades fueron consensuadas con la vecinal, el equipo del centro de salud, asociaciones de comerciantes, escuelas, entre otras instituciones barriales. De igual manera, el trabajo en territorio planteó escenarios que no pudieron ser previstos, pero se logró su abordaje por medio del análisis de las debilidades y oportunidades que hicieron posible lograr mejores resultados al momento de la intervención.

La FCM aportó el punto de vista científico a través de sus cuadros técnicos: estos compartieron algunas instancias con equipos municipales y provinciales y en otras oportunidades con todos los actores de la MTI y organizaciones barriales. Estudiantes y docentes de las disciplinas Salud Pública y Clínica Médica participaron de los equipos de trabajo en territorio, los cuales tuvieron la tarea de recorrer casa por casa para relevar la situación medioambiental y de salud individual y familiar en cuanto a la enfermedad del dengue en las zonas deli- 
mitadas previamente. Estos equipos estuvieron integrados además por personal municipal, voluntarios de la Cruz Roja, miembros del equipo de salud del barrio y vecinos que, de modo particular o por medio de una institución, habían sido convocados.

La función de los referentes territoriales (vecinales, centros de jubilados, oficinas de distrito de la ciudad, entre otras) fue estratégica, ya que brindaron información importante para la planificación y la ejecución del proyecto. Esto permitió optimizar el uso de recursos y llegar a un mayor cumplimiento de los objetivos. El rol activo de los referentes territoriales en el desarrollo de las actividades logró una mayor colaboración y predisposición por parte de los vecinos, ya que, en las zonas con mayor compromiso del referente, se consiguió acceder a trabajar en el descacharrizado asistido a un mayor número de domicilios y, además, se pudo garantizar un seguimiento del trabajo sostenido en el tiempo.

\section{La reflexión y evaluación de la intervención}

Los equipos docentes hicieron un trabajo de planificación que implicó identificar los contenidos disciplinares involucrados en la problemática, los cuales fueron transversales a varias disciplinas de la carrera de Medicina, como Salud Pública, Clínica Médica, Informática aplicada a la medicina. Los profesores pudieron anticiparse a situaciones en las diferentes instancias para abordar contenidos, pero hubo imprevistos que, con el riguroso acompañamiento docente en el desarrollo de las actividades, hicieron repensar la teoría en los nuevos escenarios.

Para la evaluación de los estudiantes se diseñó una herramienta que posibilitó reflexionar con el conjunto de los actores universitarios que participaron de las actividades. Dicha herramienta consistió en preguntas disparadoras sobre la experiencia y la puesta en práctica del contenido teórico en distintas situaciones fuera del contexto del aula. Su objetivo fue evaluar también la calidad de la experiencia de los estudiantes sobre su rol en los equipos y las relaciones con los actores intervinientes durante la práctica. Se buscó, asimismo, reflexionar sobre experiencias personales, contrastarlas con prácticas de extensión previas en un intento por hacer una valoración de las fortalezas y las debilidades que este tipo de intervenciones genera en la formación de los profesionales de la salud y el impacto que tiene en la comunidad.

Se pidió un informe por estudiante para que autoevaluara su desempeño en relación con el trabajo técnico-académico que debió realizar. Para ello, de cada actividad en la que participó, se le solicitó que calificara los siguientes puntos: posibilidad de seguir construyendo a partir de conocimientos previos, de adaptación a nuevas situaciones, la capacidad de organización del trabajo, de gestión de la información, de registro de datos y de ejecución de acciones planificadas. En ese informe, el alumno evaluó su desempeño respecto de su desarrollo personal (compromiso y actitud) así como la capacidad de escucha frente a las inquietudes expresadas por otros actores, actitud para observación y posterior análisis, capacidad para reconsiderar posiciones propias en el diálogo con otros actores y sus pares durante el desarrollo de las actividades, adaptación al territorio, capacidad para tomar decisiones y asumir el trabajo colaborativo. Se solicitó además confeccionar una matriz FODA (Fortalezas / Oportunidades / Debilidades / Amenazas) sobre su experiencia en la práctica de extensión. Y se interrogó sobre cómo se había sentido en el desarrollo de 
la propuesta y qué considero que se había aprendido. Estas consignas además sirvieron de disparadores para los talleres de reflexión.

Una vez finalizada la práctica, se convocó a todos los actores a una instancia de reflexión para analizar el trabajo realizado, evaluar la política sanitaria implementada, discutir sus fortalezas y debilidades, analizar el rol de cada una de las instituciones en el equipo y el sentido de la experiencia para cada una de ellas.

Luego de estas etapas, equipos docentes de la Facultad y del Programa de Extensión Equidad en Salud generaron instancias de reflexión con los estudiantes, con lo cual se dio la oportunidad de redefinir conceptos de salud ambiental, salud comunitaria, APS, políticas públicas en salud, epidemiología, vigilancia epidemiológica, infecciones transmitidas por mosquitos, historia clínica familiar, procedimientos y protocolos ante la aparición de casos de dengue, entre otros contenidos abordados en el contexto del aula anteriormente.

\section{El impacto del trabajo realizado}

En la experiencia se logró poner en tensión los saberes teóricos que se habían desarrollado previamente, y el territorio fue el escenario para la interacción de diferentes saberes que fueron factores de enriquecimiento de los involucrados. El vecino fue favorecido con el contenido brindado por los equipos de trabajo y los equipos de trabajo se vieron enriquecidos por conocimientos populares transmitidos por los habitantes del barrio. Surgieron métodos caseros para prevenir picaduras de mosquitos. Algunos fundados en un estilo de vida naturista y otros en conocimientos heredados de padres o abuelos. Esta tensión entre los conocimientos obligó a su discusión en instancias de puesta en común y a la evaluación, con evidencia científica, de los conocimientos recolectados en el territorio.

Como indica Milagros Rafaghelli (2016):

"Las Prácticas de Extensión de Educación Experiencial (PEEE) interpelan lo establecido en el currículo universitario convencional; promueven encuentros fuera del aula entre distintos actores e instituciones y diálogos con la autenticidad de las situaciones de la vida real. Problematizan cada campo de saber disciplinar, escuchan nuevas voces y abren nuevas preguntas". (p. 9)

Todo el equipo coincide en la importancia de este tipo de experiencias para el vínculo con diferentes actores locales y barriales involucrados en el área de la salud. Estas instancias generan trabajo en equipos que se conforman con personas con trayectorias y saberes diferentes, que se ponen en común y enriquecen no solo el trabajo en la comunidad sino a los miembros del mismo equipo.

Poder participar de las diferentes instancias que tiene una política pública como actores de la MTI local ayudó a comprender de manera práctica y tangible para el estudiante los contenidos teóricos que se abordan en la disciplina de Salud Pública de la FCM, siempre dando la importancia que tiene a cada uno de los pasos, desde la identificación y definición del problema social hasta la evaluación de los resultados. Esto permitió dimensionar la importancia que tiene la concepción de las políticas de salud cercanas a la realidad concreta y a la comunidad y la manera en que repercuten e interactúan con la salud de la población. 
Los estudiantes generaron herramientas para sortear dificultades a la hora de lograr los objetivos: consolidar el trabajo en equipo, aprender de los diferentes actores implicados y adquirir, a la vez, otras herramientas de mejor calidad en la comunicación.

El equipo de extensión coincidió en que las principales dificultades estuvieron relacionadas con el grado de compromiso de algunos de los actores involucrados, el cual en algunas oportunidades no coincidió con la agenda planteada inicialmente, causó dificultades en la operatividad y a la hora de transmitir el mensaje en algunas situaciones puntuales. En este último caso, fue fundamental que los equipos territoriales estuvieran conformados por profesionales del área de salud local, referentes territoriales y representantes de otras instituciones, para poder construir un mensaje claro que fuera entendido por el vecino. Todos coincidieron en que ese aspecto fue uno de los más interesantes a rescatar de la experiencia.

Respecto de los estudiantes, la experiencia les permitió poner en valor el trabajo comunitario en materia de prevención de enfermedades como el dengue e identificar los factores sociales que determinan la salud de las personas. Por otro lado, puso en evidencia el problema que implican para la región las enfermedades transmitidas por el mosquito y la posibilidad de abordarlas en una dinámica de trabajo interinstitucional e intersectorial en el territorio. Este tipo de intervenciones es fundamental para la formación de un profesional con bases sólidas en el primer nivel de atención, respondiendo a la estrategia de APS, que tiene una visión holística de la salud y puede entender a la persona como un ser bio-psico-social.

En el trabajo colectivo se logró recabar información importante que permitió la intervención del Estado para eliminar criaderos de mosquitos existentes en todas las zonas trabajadas. A su vez, se incorporaron más dispositivos para el monitoreo de la ovoposición, esto permite mejores herramientas a la hora de evaluar el riesgo que se encuentra el barrio ante una circulación viral.

Las instituciones que participaron hoy cuentan con equipos más capacitados, en especial la municipalidad de Santa Fe, que dispone en la actualidad de profesionales con herramientas para hacer frente a esta problemática, con una impronta de trabajo intersectorial e interinstitucional, con experiencia en el territorio, que brinda su capacidad técnica y operativa a la comunidad, ante la aparición de casos y en materia de prevención de enfermedades transmitidas por mosquitos.

El Estado hoy se encuentra más preparado en lo que refiere a enfermedades transmitidas por mosquitos; la puesta en agenda pública de la enfermedad del dengue posibilitó, a través de la MTI, la elaboración de protocolos de actuación municipales que permiten responder de manera rápida y ordenada ante la aparición de casos sospechosos, los mismos implican la coordinación con diferentes niveles del Estado y diversas áreas del gobierno local. En este sentido, se generan informes periódicos respecto a la situación local que se ponen a disposición de la MTI para evaluar políticas, implementarlas y pensar nuevas estrategias. Hay un trabajo sostenido e intensificado en los últimos años en materia de prevención de estas enfermedades haciendo foco en el autocuidado y en el ambiente y además se fortalecieron líneas de investigación en equipos locales y de la Universidad, consolidándose la participación de referentes territoriales en intervenciones referidas a la salud, junto a equipos de los centros de APS del barrio, equipos municipales, de la Cruz Roja y de la universidad. 
Los equipos de salud locales, a raíz de esta experiencia, cuentan con más y mejores herramientas para hacer frente a esta enfermedad. La incorporación de los mismos al trabajo puerta a puerta permitió el aporte de los profesionales en un contexto distinto del consultorio, donde el equipo del primer nivel de atención pudo dimensionar las dificultades que plantea la comunidad en materia de manejos de residuos o higiene en los domicilios. Eso posibilitó que los equipos de salud del barrio pudieran darse estrategias para trabajar sostenidamente en el tiempo y con las familias.

Los trabajadores del centro de salud vieron esto como una instancia donde pudieron compartir saberes y encontraron un apoyo al trabajo de todos los días, lo que sumó calidad a la tarea que se venía realizando e hizo posible dar una respuesta a mayor escala, atendiendo a la dimensión de la demanda y el contexto de brote que había acontecido. La experiencia de trabajo interinstitucional e intersectorial resultó de gran importancia para generar mejores condiciones ambientales y personales, para estar mejor preparados, y para evitar un brote de dengue la temporada estival siguiente.

\section{Conclusiones}

Se desarrollaron con éxito los operativos planificados en los distintos barrios de la ciudad de Santa Fe; se logró trabajar en las 220 manzanas previstas y se pudieron relevar 1083 domicilios. En el 21,5 \% de ellos, se ingresó a la casa y se trabajó en la descacharrización asistida; en los restantes se logró brindar información y sensibilizar respecto de la necesidad de eliminar criaderos de mosquitos. Además, se pudieron relevar datos que brindaron información detallada sobre la realidad sanitaria de cada uno de los barrios antes mencionados, de manera de disponer de más y mejores herramientas a la hora de planificar futuras intervenciones, lograr mejores resultados y una mayor optimización de los recursos.

La falta de cooperación de algunos vecinos fue una barrera importante en el trabajo. El replantear instancias previas de trabajo con ellos o buscar otras vías para la comunicación de la intervención, antes de llegar a la puerta de la casa, es importante. Se debería trabajar más intensamente y con otras estrategias que ayuden a cambiar positivamente lo ocurrido. En esta instancia es fundamental el rol de los referentes territoriales o equipos de trabajo del barrio.

Las intervenciones encontraron mejor preparados a los equipos locales de salud y a los barrios en la temporada estival siguiente, ya que el descacharrizado disminuyó el número de criaderos de mosquitos y, por ende, la probabilidad de contagio de la enfermedad ante la circulación viral.

Este tipo de experiencias abre la puerta a la generación de nuevos conocimientos que brindan herramientas a los actores que participan de ellas, lo cual suma a su formación general, ofrece diversos escenarios de aprendizaje de conocimientos estratégicos, brinda un enfoque holístico y la oportunidad de un trabajo transdisciplinario, a la vez que fortalece la formación del profesional sanitario en un perfil de APS.

Se trata de instancias que permiten aprender fuera del aula, en el contexto real; dan la posibilidad de poner a prueba las habilidades y los conocimientos teóricos que se poseen, de fortalecerlos y de evaluar sus consecuencias, identificando nuevos problemas y fijando prioridades en cuanto a las urgencias de su solución. 
La MTI resulta un espacio esencial para fomentar la participación activa de la comunidad en la toma de decisiones, en la construcción de una agenda y en la gestión y evaluación de las políticas públicas de salud. Esta participación es fundamental para el desarrollo y el bienestar de la comunidad.

En este sentido, la práctica de la intersectorialidad brinda una mayor capacidad de respuesta en cuanto a cantidad, calidad y dinamicidad para enfrentar los problemas vinculados a la salud, bienestar y calidad de vida, ya que convoca a la intervención coordinada de instituciones representativas de la comunidad.

La MTI se configura como un escenario para el aprendizaje en el contexto de la acción social, de vínculo con diversos actores, pero también es una herramienta que permite a la FCM, y a la UNL en general, ser protagonistas en la construcción de la agenda pública en materia de salud y ofrece la oportunidad a equipos docentes y estudiantes de participar en la planificación, ejecución y evaluación de políticas públicas locales mediante el aporte del conocimiento que la academia genera.

Crear este tipo de espacio para el vínculo de los actores que se interesan por la calidad de vida de las personas implica democratizar el acceso a la información, a la toma de decisiones, a la planificación y la ejecución de estrategias, lo cual redunda en beneficios para la comunidad, propicia el autocuidado de la salud y la prevención de enfermedades, en este caso, las transmitidas por mosquitos.

El espacio en la MTI, liderada por el gobierno local, da a la Facultad un lugar especial para la gestión de políticas públicas en salud y favorece una presencia territorial que brinda oportunidades, que contribuye a la educación para la ciudadanía y a la formación ética de quienes forman parte del trabajo en equipo y sus destinatarios. También facilita a los universitarios, dada la naturaleza de las actividades que se realizan desde la MTI, el conocimiento del contexto comunitario y social, al tiempo que admite brindar servicios de valor positivo en respuesta a demandas externas a la universidad.

\section{Referencias bibliográficas}

Baró, S. (s.f.). Mesa de Trabajo Intersectorial. Ministerio de Salud de la Nacion, http://www.msal.gob.ar/images/stories/bes/graficos/0000001053cnt-2014-01_mesa-trabajo-intersectorial.pdf

Díaz Quiñones, J.; Valdés Gómez, M. y Boullosa Torrecilla, A. (2016). El trabajo interdisciplinario en la carrera de medicina: consideraciones teóricas y metodológicas. MediSur, 14(2), 213-223.

Gofin, J. y Gofin, R. (2012). Salud Comunitaria Global. Principios, métodos y programas en el mundo. Elservier Masson.

Menéndez, G. y Tarabella, L. (2016). El aprendizaje experiencial: una práctica de innovación que se afianza en la Universidad Nacional del Litoral. +E: Revista de Extensión Universitaria, 6(6), 96-103. https://doi. org/10.14409/extension.v1i6.6317

Ministerio de Salud de la Nación (diciembre de 2014). Programa Nacional de Municipios y Comunidades Saludables. Boletin Virtual N 6. Argentina. http://www.msal.gob.ar/images/stories/bes/graficos/0000001216cnt-boletin_n6_diciembre_2014_salud_comunitaria.pdf

Ministerio de Salud de la Provincia de Santa Fe (26 de noviembre de 2013). Gobierno de la Provincia de Santa Fe. http://www.santafe.gov.ar/index.php/web/content/download/184031/897173/file/bi_14_ 
Organización Mundial de la Salud (OMS) (1998). Promocion de la Salud Glosario. OMS.

(s/f.). www.who.int. https://www.who.int/social_determinants/es/

Rafaghelli, M. (2016). Las Prácticas de Extensión de Educación Experiencial como oportunidad para integrar docencia y extensión. +E: Revista de Extensión Universitaria, 6(6), 8-15. https://doi.org/10.14409/extension. v1i6.6308

Rovere, M. (2006.). Planificación estratégica de recursos humanos en salud. Organizacion Panamericana de la Salud. 